\title{
Percepción, intencionalidad y lenguaje en el aristotelismo del siglo XIII
}

\author{
Jörg Alejandro Tellkamp \\ Universidad Nacional de Colombia
}

In this paper I wish to argue that in $13^{\text {th }}$ century epistemology, the perception of the physical world did include the apprehension of complex states of affairs which could also be grasped by some animals. By discussing Avicenna's and Aquinas' points of view regarding the concept of sensibile per accidens and the mental faculties of perception (vis aestimativa and vis cogitativa), I conclude that the perceptual act of discerning complex states of affairs is based upon a background knowledge in which language is a non-essential feature.

\section{1. 'Introducción'}

En el centro del "giro lingüístico" del siglo XIV desde Guillermo de Ockham se encuentra el supuesto de que los estados mentales de alto nivel, es decir los estados intencionales y cognitivos, solamente pueden darse, en cuanto que se disponga de un aparato conceptual y, pcr ende, de la capacidad de formar proposiciones ${ }^{2}$. La percepción

\footnotetext{
1 Versiones anteriores se leyeron en el XI Congreso de Filosofia Medieval en agosto de 2002 en Porto y en primer Taller de Filosofia Antigua realizado por el Instituto de Investigaciones Filosóficas de la UNAM en julio de 2003. Agradezco a los participantes sus valiosos comentarios, pero sobre todo a Luis Xavier López Farejeat por su comentario minucioso y critico.

2 Véase PERLER, D.: Theorien der Intentionalität im Mittelalter, Frankfurt/M: Klostermann 2002, p. 362 con respecto al punto de vista ockhamiano: "Der Akt, mit dem ein Gegenstand erfasst und verstanden wird, ist also nicht etwas
} 
del mundo exterior parecería calificar como uno de estos estados y procesos mentales de alto nivel, en el sentido en que, por ejemplo, ver un avión u oír un discurso requiere de ciertas condiciones que permitan decir que "veo un avión" u "oigo un discurso". El problema que desarrollaré es expresado con suficiente. agudeza por Cyrille Michon: "Lo que $x$ ve, lo ve como algo, bajo una descripción. ¿Significa esto que toda percepción incluye un juicio, una creencia o que los aspectos bajo los cuales los objetos son percibidos son pensamientos expresados por una oración proposicional que la sigue?"3.

A diferencia de la 'reducción' de episodios perceptivos' a un análisis de proposiciones, tal como fue desarrollada por Ockham, existe la propuesta desde los principios del siglo XIII - con la recepción de los escritos de Avicena y de Averroes - de explicar los procesos perceptivos a partir de algo parecido a una psicología cognitiva; utilizando modelos orgánico-fisịológicos de-la percepción para elucidar la producción de la cognición sensorial. De esta manera se llega a una teoría global de la percepción que logra establecer los criterios que explican por qué los animales superiores ${ }^{4}$, incluyendo a

Präkonzeptuelles, das erlaubt, in einem weiteren Schritt einen Begriff 'für den Gegenstand zu bilden. Der Akt selbst hat schon eine begriffliche Struktur".

${ }^{3}$ Michon, C.: "Intentionality and Proto-Thoughts", Dominik Perler (ed.), Ancient and Medieval Theories of Intentionality, Leiden-Boston-Köln: Brill 2001, p. 328s. Hablar de pensamiento en conexión con procesos de la percepción podria parecer exagerado, porque se tendría que suponer que los animales piensan, cuando percibien. Para dar cuenta tanto de la complejidad de los contenidos perceptivos; como de su carácter no-proposicional, sugiere $\mathrm{C}$. Michon llamar esos fenómenos mentales "proto-pensamientos. En todo caso no se pretende sugerir que estos protopensamientos sean racionales, en el sentido de una atribución proposicional o que la posesión de conceptos determine necesariamente las creencias que los 'animales y seres humanos tienen acerca del mundo. De hecho existen intentos recientes para argumentar, desde la perspectiva actual de la Filosofia de la Mente, que "language is not necessary for thought"; sobre todo para poder analizar los estados mentales complejos de los animales superiores y los niños; cf. BERMidDEZ, J. L.: Thinking without Words, Oxford-Nueva York: Oxford University Press 2003, p. 5.

${ }^{4}$ Los animales superiores o perfectos son aquellos que disponen de un aparato cognitivo completo, es decir de los cinco sentidos exteriores y de los siguientes sentidos interiores: sentido común, fantasía y/o imaginación, memoria y vis aestimativa 0 cogitativa. 
los animales racionales, llegan, de una u otra -manera,' $a$ un conocimiento : perceptivo complejo del mundo, es decir a un conocimiento intencional del mismo.

En general, la percepción, que respecto del conocimiento intelectivo es de "bajo" nivel; se puede concebir como un tipo de creencia: o proto-pensamiento acerca del mundo físico que siempre también es intencionals . Cuando Pedro ve una casa, él no solamente ve un objeto material de tales o cuales características cualitativas y de determinado aspecto; él 'más' bien ve una casa. Ver una casa; es decir conocer perceptivamente un objeto material particular de determinado tipo, constituye un conocimiento complejo; por cuanto que "casa" no es algo percibido simpliciter, es decir : que "el significado de un objeto determinado de ladrillos y cemento no es un fenómeno que transforme el apàrato sensitivo; esto sólo lo hacen las características cualitativas del objeto, como es por ejemplo el color. De esta manera hay què distinguir dos aspectos fundamentales en la percepción de un objeto: (1) el aspecto esencial, que altera causalmente el aparato sensitivo y (2) el aspecto per accidens, que lleva a que el animal perciba una cosa material bajo un aspecto cognitivo y no-trivial. Esta no-trivialidad de los contenidos perceptivos aplica al conocimiento sensorial animal: "cuando' una oveja ve un lobo, para usar el ejemplo de Avicena, no solamente ve un objeto material gris, 'sino que ve en él un enemigo de su

${ }^{5}$ Cuando hablo de intencional, es importante no confundir esta noción con la que fue 'propagada por F. Brentano; quien piensa que los fenómenos mentales son relacionalés y que su correlato es un objeto intencional que no es idéntico con el objeto material, sino con un objeto mental. Es en este sentido que Brentano habla de la inexistencia intencional; cf: BRENTANO, F.: Psychologie vom empirischen Standpunkt, vol...1, OsKAR, K. (ed.), Hamburgo: Meiner 1973, p.:124s. Véase también Perler: Theorien der Intentionalität pp. 1-11.Como se verá en este texto, Tomás de Aquino está de acuerdo con la tesis de la intencionalidad como fenómeno relacional, pero difiere, porque no piensa que ésta sea un fenómeno netamente mental, sino que se basa de manera fundamental en los procesos causales, necesarios para estimular el aparato sensitivo. Dicho de otra manera: si una cosa determinada no ejerciera su influencia causal, por ejemplo, sobre el ojo, entonces no llegariamos a percibir esa cosa como, por ejemplo, una casa. Brentano, quien parte en lo esencial de la teoria del franciscano Pedro Aureoli (c. 1280-1322), descarta un vínculo causal entre la cosa material y el ser cognitivo. 
naturaleza, y esto parecería significar que tiene algún tipo de creencia, por cierto preconceptual, acerca del objeto que percibe ${ }^{6}$. A ambos, Pedro y la oveja, el mundo físico aparece de una manera significativa.

Esta forma de percibir el mundo de manera significativa es descrita por Avicena y por muchos de los pensadores aristotelizantes del siglo XIII con el concepto de intentio. En lo que sigue trataré de establecer un acercamiento a la psicología de la percepción intencional partiendo de las disquisiciones del filósofo persa, para luego ampliar estas observaciones con un análisis más detallado de los sensibles por accidente según Tomás de Aquino, quien junta esta teoria aristotélica con la noción aviceniana de intentio.

Mi tesis será que la creación de un conocimiento perceptivo intencional depende esencialmente del funcionamiento de las facultades del aparato cognitivo, sobre todo de los así llamados sentidos internos o interiores, junto con un conocimiento antepuesto, bien instintivo (en animales) o bien teórico (en seres humanos). La estructura de este aparato es isomórfico en los animales superiores y en los seres humanos; es decir entre los animales no racionales y los racionales existe una identidad estructural del aparato perceptivo que lleva a un conocimiento sensible comparable. El conocimiento de las intentiones se basa entonces en la fisiología y la psicología de la mente. Por supuesto habria que preguntarse, qué papel juega el lenguaje en este esquema, y yo trataré de dar algunas pistas de por qué el lenguaje queda relegado a un segundo plano no esencial. Con todo, en los seres humanos, las capacidades racionales, y por ende lingüísticas, permiten llegar a un grado mayor de complejidad en el conocimiento sensible intencional, en cuanto que este se basa en el mencionado conocimiento de fondo que se activa siempre cuando se da un estímulo apropiado.

${ }^{6}$ Cf. Avicena: Liber De Anima seu Sextus De Naturalibus, vol. 2, S. van Riet (ed). Louvain/Leiden: Éditions Orientalistes/Brill 1968, p. 7, 82-86. 


\section{Avicena}

No cabe duda que la discusión acerca de la intencionalidad en el conocimiento comienza con el pensador persa Ibn Sina o Avicena (c. 980 - c. 1038) - así su nombre latinizado - cuyo Liber sextus de naturalibus seu de anima, traducción latina de la Shifa, fue ampliamente leído y discutido en el siglo XIII.

Llama la atención que Avicena, al discutir la noción de intentio, la distingue en primer lugar de la aprehensión de una forma y que, en segundo lugar, la analiza principalmente en conjunción con un análisis de aquellas facultades cognitivas que aprehenden $a b$ intus, o sea los sentidos internos ${ }^{7}$. Véase la siguiente cita:

La diferencia pues entre aprehender una forma y aprehender una intención es esta: la forma es aquella que es aprehendida simultáneamente por el sentido interno y externo, pero el sentido externo la aprehende primero y después la remite al sentido interno, así como cuando la oveja aprehende la forma del lobo, es decir su forma, afección y color. Pero el sentido externo de la oveja la aprehende primero y después el sentido interno. La intención, en cambio, es lo que el alma aprehende del objeto sensible, aunque no la aprehenda primero el sentido exterior, tal como la oveja aprehende la intención que tiene del lobo, es decir que es por qué lo debe temer y rehuir, aunque esto no lo aprehenda con los sentidos de ninguna manera. Sin embargo, lo que el sentido externo y

\footnotetext{
${ }^{7}$ AviCENA: De an. 1, 5 p. 83, 57ss: "Differentia autem inter apprehendere formam et apprehendere intentionem est haec: quod forma est illa quam apprehendit sensus interior et sensus exterior simul, sed sensus exterior primo apprehendit eam et postea reddit eam sensui interiori, sicut cum ovis apprehendit formam lupi, scilicet tiguram eius et affectionem et colorem, sed sensus exterior ovis primo apprehendit eam et deinde sensus interior; intentio autem est id quod apprehendit anima de sensibili, quamvis non prius apprehendat illud sensus exterior, sicut ovis apprehendit intentionem quam habet de lupo, quae scilicet est quare debeat eum timere et fugere, quamvis non hoc apprehendat sensus ullo modo. Id autem quod de lupo primo apprehendit sensus exterior et postea interior, vocatur hic proprie nomine formae; quod autem apprehendunt vires occultae absque sensu, vocatur in hoc loco proprie nomine intentionis". Todas las traducciones son mias.
} 
después el interno aprehenden primeramente del lobo; aqui se llama propiamente con el nombre de forma. No obstante, lo que aprehenden las facultades ocultas sin los sentidos; se llama en este lugar propiamente con el nombre de intención ${ }^{8}$.

Partiendo de esta cita; quisiera recalcar los siguientes elementos. La aprehensión de una forma consiste enila.captación de los aspéctos meramente cualitativos y cuantitativos (colores, el aspecto exterior etc.) Esta captación comienza, en un sentido causal, con los sentidos externos; para luego pasar a los sentidos internos. Este tipo de aprehensión corresponde a lo que Aristóteles llama los sensibles esenciales. Los sentidos externos de la oveja captan estas facetas, y sin embargo, llegan a un conocimiento acerca de lo que el lobo 'significa'. La significación es expresada por el concepto de intentio. Esta remonta a un objeto sensible, pero no es captada directamente por medio de la actividad de los sentidos externos, porque no forma parte de aquellos atributos de un objeto físico que suelen producir efectos. en otros objetos: De esta manera se hace necesaria la actividad: de los sentidos internos, que Avicena llama 'facultades ocultas', sobre todo la de la así llamada vis o virtus aestimativa, a la que haré alusión más adelante.

Pero, ¿qué son las intentiones?. Dag N. .Hasse propone interpretarlas como atributos connotacionales ${ }^{9}$. Las intenciones, aunque :no directamente captadas por. los sentidos externos, son atributos' de los objetos físicos', y no exclusivamente de la mente. De esta manera se.trata de. rasgos que poseen una existencia real $y$, sin embargo, son captados únicamente por los sentidos ocultos, o sea internos, que, por medio de procesos mentales y de la aprehensión de las formas sensibles, los revelan. La razón por la que las intenciones no son directamente captadas por los sentidos externos, está en que ellas no forman directamente parte del proceso causal como "las formas sensibles (colores, sonidos etc.). El ojo, por ejemplo, capta

\footnotetext{
8 AVICENA: De an. I, 5 p. 86, 93-106.

9. Dag. Hasse, N.: Avicenna's De anima in the Latin West, The Formation of a Peripatetic Philosophy of the Soul 1160-1300, Londres-Turin: Warburg. InstituteNino Aragno Editore 2000, p. 132ss.
} 
las formas sensibles, es decir la extensión de las cosas, sus colores; sonidos etc., pero no capta directamente su 'significado'. Este aspecto queda relegado a una instancia ulterior que posee la capacidad de adscribir a lo captado la intencionalidad que de hecho tiene.

La oveja, entonces, no huye del lobo, porque a manera de reflejo sienta pánico; sino porque percibe un hecho en el mundo, i.e. la hostilidad que acompaña a la captación de los rasgos formales del lobo ${ }^{10}$ : El concepto de instinto es entonces mucho más amplio que el de una mera relación entre estímulo y reacción, porque incluye una aprehensión activa de la oveja con respecto a los aspectos generales del objeto percibido. Como dice Hasse:

Me parece que es una teoría relativamente fuerte acerca del instinto, porque trata de explicar cómo los animales y: los seres humanos no solamente reaccionan inmediatamente, sino que perciben las causas reales para sus reacciones que no "'pueden ser captadas por medio de la percepción sensorial ${ }^{1 !}$.

Este punto me lleva a cómo se captan las intenciones que no son propiamente percibidas por los sentidos exteriores: 'Para ello es indispensable introducir 'el concepto de virtus aestimativa, que es una facultad de la mente que se localiza en el ventrículo central del cerebro. Entre sus varias funciones ${ }^{12}$ hay que destacar aquella que permite la adsçripción de intencionalidad a un objeto percibido. Esta función es posible, porque la virtus aestimativa incluye un tipo de conocimiento de fondo, que en los animales eš instintivo, y que en los seres humanos ès moldeado por la razón: Este background knowlédge, como dice Searle ${ }^{13}$, es de naturaleza disposicional, por lo que siempre es activado, cuando se da un estímulo sensorial

\footnotetext{
${ }^{10} \mathrm{Cf}$. Hasse op.cit. p. 136.

11 Ibid.

12 Hasse op.cit. p. 138 .

13 Véase SEARLE, J.: Intentionality. An Essay in the Philosophy of Mind, Cambridge: Cambridge University Press 1983, pp. 37-78 y 141-159.
} 
adecuado. Como se verá más adelante, el background knowledge de los animales es de carácter instintivo, lo que significa que ellos poseen una estructura cognitiva innata que se "dispara" siempre cuando existe un estímulo sensorial apropiado. Esta estructura cognitiva subyacente difiere de nuevo de los procesos netamente causales, que activan los sentidos exteriores; en otras palabras, mientras que las características accidentales de las cosas son captadas a manera de datos sensoriales crudos, las intenciones no son propiamente captadas por los sentidos externos, sino que se manifiestan una vez un determinado color o sonido haya sido captado. La diferencia entre la captación de los datos sensoriales crudos y la aprehensión de las intenciones constituye la diferencia entre "sensación" y "percepción".

Pero las cosas que no son sensibles por su naturaleza, son tales como la enemistad y la maldad y que de suyo se rehuyen, [es decir] lo que la oveja aprehende de la forma del lobo, y en general la intención que la hace huir de él. También aprehende la concordancia con sus congéneres y generalmente toda intención con la que se alegra con ellos. Estas son las cosas sensibles que el alma aprehende de tal manera que los sentidos no le muestren algo de ellas. Por tanto, la facultad por la que estas cosas son aprehendidas [es decir la imaginativa - J.A.T.] es una facultad distinta y es llamada aestimativa ${ }^{14}$.

Según Avicena, se pueden distinguir tres tipos de conocimiento de fondo que se manifiestan en la virtus aestimativa ${ }^{15}$. (1) El primero, por "clemencia divina", se deriva directamente de la constitución fisica de los animales, en cuanto que puedan reaccionar a un estímulo sin una aprehensión determinada del mundo exterior. Con

\footnotetext{
14 AviCENA: De an. IV, 1 p. 7, 82-88: "Sed quae non sunt sensibiles ex natura sua, sunt sicut inimicitiae et malitia et quae a se diffugiunt quam apprehendit ovis de forma lupi et omnino intentio quae facit eam fugere ab illo, et concordia quam apprehendit de sua socia et omnino intentio qua gratulatur cum illa: sunt res quas apprehendit anima sensibilis ita quod sensus non doceat eam aliquid de his; ergo virtus qua haec apprehenduntur est alia virtus et vocatur aestimativa".

${ }^{15}$ AviCEna: De an. IV, 3 p. 37ssq., 19-52.
} 
esto Avicena se refiere a lo que llamariamos reflejo. (2) El segundo tipo se entrelaza con las sensaciones acerca del mundo y se da cuando el animal tiene un conocimiento innato acerca de lo nocivo o benéfico de una cosa (cautelas naturales). (3) En tercer lugar se adquiere ese conocimiento de fondo por experiencia, como cuando el perro aprende que tiene que temer los palos o las piedras. En este sentido más amplio también hay que incluir al ser humano, cuyo conocimiento de fondo sobrepasa la percepción por experiencia de los animales. Los seres humanos están en condiciones de aprehender hechos nuevos por experiencia, porque tienen la capacidad de aprender conceptos $y$ teorias que forman parte del conocimiento racional de fondo.

\section{Tomás de Aquino y los sensibilia per accidens}

Tomás de Aquino, siguiendo a Aristóteles, analiza la constitución del conocimiento sensible en diferentes pasos: comienza con los elementos básicos de la percepción, es decir con los colores, sonidos, pero también los movimiento y aspecto exterior. El siguiente paso remite a aquel tipo de conocimiento perceptivo complejo e intencional que es resumido bajo el título de sensibile per accidens.

En esta parte del presente trabajo quisiera mostrar que el isomorfismo cognitivo de animales superiores y seres humanos se debe a la identidad estructural del aparato cognitivo de los sentidos exteriores e interiores. Esto, por ende, produce resultados cognitivos comparables en cuanto a la estructura de lo percibido ${ }^{16}$. Para poder argumentar a favor de este isomorfismo estructural, trataré de mostrar que la producción de las percepciones depende esencialmente de los procesos fisicos del aparato cognitivo.

${ }^{16}$ Este es básicamente el punto de vista de SEARLE: "Animal Minds", JOHN, S.: Consciousness and Language, Cambridge: Cambridge University Press 2002, pp. 61-76; p.ej. en p. 62 . Para un análisis del conocimiento animal en el pensamiento antiguo, véase SORABJI, R.: Animal Minds and Human Morals: The Origins of the Westerm Debate. Londres: Duckworth 1993, pp. 17-49. Para un acercamiento a este tema en el pensamiento medieval, véase C. MiCHON op.cit. pp. 325-342. 
- Para llegar a este punto analizaré los elementos constitutivos de cualquier acto perceptivo, que son los siguientes: (1) los sensibles esenciales (per se) como: condiciones necesarias, mas no suficientes para la generación de sensibilia per accidens; (2) los diferentes sentidos del concepto de sensibile per accidens y (3) por último, el funcionamiento de los sentidos internos, sobre todo de la vis aestimativa (en los animales superiores) y de la vis cogitativa (en los seres humanos) como condición necesaria para la formación del conocimiento perceptivo. Solamente la unión de los procesos externos de: la:percepción con los procesos internos generan las condiciones necesarias $y$ suficientes para cualquier conocimiento intencional.

Me centraré principalmente en dos textos, pero, desde luego, existen muchos más: Summa Theologiae I q. 78 a. 3-4 y Sentencia Libiri De Anima II, 13 ${ }^{17}$. De la Summa se desprende la distinción de lọs sensibles esenciales y accidentales. En primer lugar habla Tomás de los sensibles esenciales que son el efecto en un órgano recipiente causado por las propiedades cualitativas de un objeto físico. En este punto distingue además dos tipos de sensibles esenciales los propios (sensibilia propria) y los comunes (sensibilia communia) ${ }^{18}$. En segundo lugar habla de aquellos senșibles que no son percibidos directamente, es decir los sensibles per accidens.

Los sensibles per se constituyen el fundamento necesario para cualquier actividad perceptiva, ya que son los rasgos objetivos que cualquier sentido recibe (colores, sonidos etc.). Los sensibles comunes también son captados esencialmente, pero dependen lógicamente de los sensibles propios. Toda actividad de la percepción es acompañada por la captación simultánea de los aspectos categoriales de un objeto físico, como lo son movimiento,

17 Cfr. Sancti Thomae de AQuino: Opera Omnia iussu Leonis XIII P.M. edita. Summa Theologica, Tomus IV, Sentencia Libri De Anima, Tomus XLV,1, Roma/París: Commissio Leonina/Vrin 1984.

18 La terminología es introducida por Tomás en In DA II, 13 p. 118, 18-23: "Sensibilia uero tribus modis dicuntur, uno quidem modo per accidens et duobus per se, quorum uno dicuntur sensibilia illa que propria sunt singulis, alio modo dicuntur sensibilia illa que communiter senciuntur ab omnibus sensibus". . 
reposo, número; aspecto $y$ tamaño ${ }^{19}$. Estos sensibles se llaman comunes, porque representan aspectos perceptibles que pueden ser captados por uno o más sentidos exteriores. El tamaño de una cosa, por ejemplo, puede ser captado por el sentido del tacto, tanto como por el sentido de la vista.

Tomás parece pensar que estos sensibles constituyen "las condiciones necesarias, mas no suficientes, para cualquièr acto complejo e intencional. Sin haber captado previamente (en el sentido de anterioridad lógica) el color de una manzana, no podria darse la percepción de una manzana. Lo-mismo aplica al tamaño;'al aspecto exterior: de la manzana, etc. Sin. embargo, estos componentes cualitativos $y \cdot$ cuantitativos en si mismos : no. constituyen conocimiento perceptivo alguno, porque en la captación de por ejemplo 'rojo' y 'redondo' no remiten aún a un objeto determinado como sí lo haría la captación de 'manzanà'. De todas maneras; los sensibles per. se representan elementos constitutivos y necesarios de ese conocimiento complejo y estructurado que permite referirse a cosas particulares.

La percepción en su sentido más amplio puede ser concebida como un tipo de creencia acerca del mundo exterior. Como tal, "se'puede pensar que reposa sobre algún tipo de teoría explícita o implícita acerca del mundo fisico, el ya aludido conocimiento de fondo (background knowledge). Evidentemente esto no significa que, cuando un pájaro toma un palillo como elemento para la construcción de un nido, tenga que disponer de un concepto sobre la naturaleza del palillo y que adicionalmente tenga en mente un plano de construcción para nidos. La "teoría" implícita:que maneja el pájaro es instintiva; la facultad orgánica del 'cerebro; la vis aestimativa, hace que el pájaro vea algo como algo ${ }^{20}$. Esta capacidad de ver el palillo como un elemento para la construcción de nidos es lo se que podría calificar como un sensible accidental. Estos no son captados propiamente como los sensibles esenciales, es decir que no

19 Véase TOMÁs de AquiNo: In De An. II, 13 p. 119, $46-48$.

20 Véase WHiTE, L: "Instinct and Custom", ${ }^{\prime}$ The Thomist LXVI (2002), pp. 577. 605. 
forman directamente parte de la causalidad que permite vincular al objeto con el órgano. Los sentidos internos - sobre todo la vis aestimativa y la vis cogitativa - juegan, no obstante, un papel causal propio para producir los sensibles accidentales.

En la Filosofia Moderna, pero también en muchas corrientes del pensamiento antiguo, el conocimiento perceptivo fue visto como un tipo inferior y defectuoso de conocimiento. Parece que Tomás opone a esta visión una epistemología naturalizada, en la cual los diferentes tipos de conocimiento, tanto perceptivo como racional, se basan en el funcionamiento del aparato sensorial. Esta estrategia "de abajo hacia arriba" (bottom-up strategy), en palabras de. Fred Dretske, significa que es necesario examinar en primer lugar el contenido perceptivo para luego analizar el conocimiento de orden superior, como lo es el conocimiento intelectivo ${ }^{21}$. Mientras que los sentidos externos realizan una labor de discernimiento con respecto a sus objetos propios, los sentidos internos conducen a aquella forma de conocimiento perceptivo rico e intencional que se llama sensibile per accidens. Dos preguntas obvias surgen en este contexto: ¿de qué tipo de conocimiento perceptivo se trata? ¿Cómo se constituye?

El texto de la translatio nova de Moerbeke del De anima 418a20ssq de Aristóteles no es muy específico lo que la naturaleza de estos sensibles respecta:

Efectivamente se habla de sensible accidental, como cuando Diares es blanco. Por cierto, esto es captado accidentalmente, porque lo blanco está en aquello que se capta. Por ende, nada es afectado por el objeto sensible en cuanto que es $\mathrm{tal}^{22}$.

${ }^{21}$ Véase DrETSKE: “Two Conceptions of Knowledge: Rational vs. Reliable Belief”, Fred Dretske, Perception, Knowledge, and Belief, Selected Essays, Cambridge: Cambridge University Press 2000, pp. 80-93, aqui p. 80.

22 Tomás de Aquino: In De An. II, 13 p. 118: "Secundum accidens autem dicitur sensibile, ut si album sit Diarris: secundum accidens enim hoc sentitur, quoniam. accidit albo hoc quod sentitur. Vnde nichil patitur, secundum quod huiusmodi est, a sensibili". 
Evidentemente lo que es captado per accidens, no es captado esencialmente. Esto significa que existe un cierto rango de propiedades que no son captadas esencialmente, ya que no están directamente involucradas en la historia causal que une el objeto físico con el órgano sensorial. Entre las propiedades perceptibles de Diares se encuentra su atributo sensible más destacado, su blancura, pero no su ser Diares.

Tomás, al comentar este texto, introduce tres nociones de per accidens. Después de haber establecido la diferencia entre los sensibilia comunia, que pueden ser concebidos como per accidens en relación con los sensibilia propria, y los sensibilia per accidens en su sentido propio, Tomás pregunta cuáles son las razones para decir que algo es un sensibile per accidens ${ }^{23}$.

En primer lugar, y por razones ontológicas, es necesario que el sensibile per accidens sea accidental con respecto a lo que es captado esencialmente. Asi, por ejemplo, la blancura es un accidente del ser humano. En segundo lugar se añade un elemento importante: para poder decir que algo haya sido captado per accidens, es necesario que haya sido aprehendido por un ser con la capacidad de sentir y percibir ${ }^{24}$. Con este punto se introduce una condición significativa: los sensibilia per accidens requieren un punto de vista cognitivo de alguien o algo, es decir la aptitud de percibir un objeto fisico bajo un punto de vista intencional. Al hablar de. 'punto de vista cognitivo', me refiero a que Tomás piensa que el concurso de las facultades cognitivas es necesario para alcanzar el conocimiento de las intenciones. Sin estas facultades no se llegaria a ningún conocimiento accidental de objetos sensibles. Partiendo de la necesidad de un punto de vista cognitivo, la percepción accidental puede ser entendida de tres maneras.

(1) La primera refiere a la capacidad que posee un sentido exterior para captar la cualidad que es esencial de otro sentido exterior. Así

\footnotetext{
${ }^{23}$ Tomás de Aquino: In De An. II, 13 p. 120, 161-163.

24 TOMÁs DE AQUino: In De An. II, 13 p. 120, 167sq: “[...] Requiritur quod sit apprehensum a senciente [...]".
} 
se puede decir que la potencia visual "ve" la dulzura de una galleta. La dulzura, sin embargo, es accidental con respecto al objeto propio de la visión, es decir el color ${ }^{25}$. Tomás anota que esta forma de sensación accidental no se puede concebir como una forma válida de conocimiento perceptivo.

(2) El segundo sentido de sensación accidental se deriva de la participación del intelecto en los procesos de percepción. De acuerdo al objeto propio del intelecto, la percepción capta en todos sus actos rasgos universales en el ámbito de lo sensible: Sin embargo, no toda aprehensión de universales en el ámbito de lo sensible representa un sensibile per accidens, sino solamente aquel sensible que es "aprehendido inmediatamente por el intelecto cuando surge la cosa captada"26. Un ejemplo puede ilustrar este punto: cuando yeo caminar o hablar una persona, $\mathrm{mi}$ intelecto aprehende inmediatamente que ese ser vive. Por tanto, yo percibo per accidens la vida de ese ser. No es del todo claro lo que este tipo de percepción per accidens: significa; pero Tomás aparentemente piensa "que, cuando una cosa. es : percibida bajo un aspecto particular (por ejemplo: persona $X$. camina), el intelecto capta el aspecto universal que forma parte de esta : cosa particular aprehendida. $\mathrm{Al}$ añadir el adverbio statim, : parece además :que Tomás se refiere a verdades analíticas que son captadas cuando un aspecto o cosa percibida incluye necesariamente otro aspecto, así como un círculo particular incluye necesariamente el aspecto de la redondez; es decir que pertenece universalmente a la categoria de la circularidad ${ }^{27}$.

(3) Esto me lleva al tercer sentido cognitivo de sensibile per accidens; del cual Tomás dice que es aprehendido como en un individuo (in singulari). Por ejemplo; al ver un objeto coloreado, "veo este hombre o este animal",28, es decir que al ver un objeto

${ }^{25}$ TOMÁs DE AQUINO: In De An. II, 13 p. 120, 175ssq.

26 ToMÁs DE AQUINo: In De An. II, 13 p. 121, 186sq.: "[...] Quod statim ad occursum rei sensae apprehenditur intellectum".

27 Véase tạmbién.Tomás de Aquino: Su. Theol. I q. 12 a. 3 ad 2.

${ }^{28}$ Tomás de Aquino: In De An. II, 13 p. 121, 192sq. 
blanco, también se ve que se trata de Diares a quien corresponde esta característica cualitativa, es decir la de ser blanco.

L'a explicación de esta percepción intencional se halla en la constitución fisiológica y mental de los animales superiores, como los seres humanos, lobos y ovejas. Tomás dice:

Por esto, ya que la potencia sensitiva es el acto del órgano corpóreo; es necesario que otra potencia reciba las especies de los objetos sensibles y que las conserve. Por el otro lado hay que considerar que, si el animal solamente se moviera debido a lo deleitable y lo aflictivo perceptible, seria necesario asumir en el animal solamente la aprehensión de las formas que percibe el sentido, con las cuales se deleita.y [de las que] rehuye. Pero es necesario que el animal busque algunas cosas o las rehuya, no solamente porque son convenientes o no convenientes para la sensación, sino también debido a otras comodidades y utilidades 0 perjuicios, como cuando la oveja, que ve al lobo, huye; no debịdo a la fealdad del color o de su aspecto, sino porque es como si fuera un enemigo natural. De manera similar el ave recoge el palillo, no porque deleite sus sentidos, sino porque es útil para construir nidos. Es entonces necesario para el. animal que perciba estas intenciones que no son percibidas por el sentido exterior ${ }^{29}$.

29 Tomás de Aquino: Su. Theol. I q. 78 a. 4 c.: "Unde, cum potentia sensitiva sit actus organi corporalis, oportet esse aliam potentiam quae recipiat species sensibilium, et quae conservet. Rursus considerandum est quod, si animal moveretur solum propter delectabile et contristabile secundum sensum, non esset necessarium ponere in animali nisi apprehensionem formarum quas percipit sensus, in quibus delectatur aut horret. Sed necessarium.est animali ut quaerat aliqua vel fugiat, non solum quia sunt convenientia vel non convenientia ad sentiendum, sed etiam propter aliquas alias. commoditates et utilitates, sive nocumenta, sicut ovis videns lupum venientem fugit, non propter indecentiam coloris vel figurae, sed quasi inimicum naturae; et similiter avis colligit paleam, non quia delectet sensum, sed quia est utilis ad nidificandum. Necessarium est ergo animali quod percipiat huiusmodi intentiones, quas non percipit sensus exterior". 
La percepción de la intención de un objeto físico es (1) un evento que no surge simplemente como consecuencia de causas naturales y (2) por ende tiene que ser incluida en una teoria de las facultades cognitivas que (3) permitan explicar cómo la oveja "juzga" que el lobo es un enemigo natural o cómo alguien cree que es Diares el que se está acercando.

La percepción de las intenciones no es un evento natural que surge con la captación de cualidades y cuantidades sensibles. Esto significa que las intenciones sensibles, por ejemplo percibir al lobo como un enemigo natural, requiere un elemento adicional. Este elemento es el de los sentidos internos, y en particular el de la vis aestimativa o cogitativa. Tomás asume que estas facultades están localizadas' en el cerebro (in media parte capitis), aunque en general no se muestra muy interesado en los detalles fisiológicos del asunto y más bien parece asumir que las opiniones de Avicena y Averroes al respecto son correctas. ${ }^{30}$

Con la ayuda de la vis aestimativa o cogitativa algo es captado bajo un aspecto intencional que no es aprehendido naturalmente. Pero, ¿qué conduce a la aprehensión de estas intenciones? A esto Tomás responde que la vis aestimativa o cogitativa ejercen sus respectivas funciones sobre la base de estructuras cognitivas subyacentes (background knowledge). En el caso de la vis aestimativa la estructura cognitiva subyacente pertenece a un conjunto de respuestas innatas o adquiridas a ciertos estímulos, respuestas que se podrian subsumir bajo el título de 'instinto' ${ }^{11}$. Estas respuestas son naturales en el sentido en que no se encuentran

${ }^{30}$ Véase ToMAS DE AQUino: In Sent. IV d. 50 q. 1 a. 1 ad 3; Su. Theol. I q. 78 a. 4 c. También Averroes: Commentarium Magnum in Aristotelis De Anima Libros, S. Crawford (ed). Cambridge (Mass.): The Medieval Academy of America 1953, p. 415, 49-51: "[...] Virtus ymaginativa est in anteriori cerebri, et cogitativa in medio, et rememorativa in posteriori". AvicENA: De an. I, 5 p. 89, 48-52: "Deinde est vis aestimationis; quae est vis ordinata in summo mediae concavitatis cerebri, apprehendens intentiones non sensatas quae sunt in singulis sensibilibus, sicut vis quae est in ove diiudicans quod hoc lupo est fugiendum, et quod huius agni est miserendum [...]".

${ }^{31}$ Véase L. WHITE op.cit. 
sujetas a la deliberación del animal, sencillamente porque los animales no racionales no poseen la capacidad propiamente racional de deliberar ${ }^{32}$. Un animal percibe la intentio de algo únicamente en cuanto que es "el término o el principio de alguna acción o pasión"33. Esto parece significar que la vis aestimativa permite que los animales superiores reaccionen adecuadamente a su entorno y que reaccionen frente a él para garantizar su supervivencia ${ }^{34}$.

El análisis de la vis cogitativa, que en el pensamiento de Tomás es el correlato humano de la vis aestimativa, es más complejo, porque en él el intelecto juega un papel importante, a pesar de que Tomás nunca aclare cómo el intelecto inmaterial pueda ejercer una influencia sobre un sentido interno material. En este sentido, el isomorfismo de la percepción animal y humana solamente aplica a la estructura orgánica del conocimiento perceptivo y la similitud de los contenidos perceptivos, pero no a su complejidad. Mientras que la vis aestimativa se ocupa exclusivamente de la aprehensión de una intención con fines prácticos, la vis cogitativa aprehende un objeto "sub natura communi". Esta es una diferencia importante de la vis cogitativa con respecto a la vis aestimativa, porque introduce aspectos racionales, que no se encuentran en el conocimiento animal.

La estructura cognitiva subyacente de los animales superiores para percibir el mundo físico de manera estructurada e intencional es, como se ha insinuado, de naturaleza instintiva. En los seres humanos, sin embargo, el instinto no juega un papel predominante con miras a adquirir un conocimiento complejo. Esta "carencia" exige un sustituto que encontramos en la participación del intelecto en los procesos de percepción. Esta participación es la razón por la

32 Tomás de Aquino: Su. Theol. I q. 59 a. 3 c.: "Respondeo dicendum quod quaedam sunt quae non agunt ex aliquo arbitrio, sed quasi ab aliis acta et mota, sicut sagitta a sagittante movetur ad finem. Quaedam vero agunt quodam arbitrio, sed non libero, sicut animalia irrationalia, ovis enim fugit lupum ex quodam iudicio, quo existimat eum sibi noxium; sed hoc iudicium non est sibi liberum, sed a natura inditum". También $S u$ Theol. I q. 81 a. 3 c. and q. 81 a. 1 c.

33 Tomás de Aquino: In De An. II, 13 p. 122, 213sq.

${ }^{34}$ Aqui no podré dedicarme a este asunto; la relación de la cogitativa con el ámbito de lo práctico es ampliamente discutido por L. WHITE op.cit. 
que Tomás equipara el término de vis cogitativa con el de ratio particularis. Su función básica es la de juntar y captar la intenciones de cosas particulares; es collatiua intentionum indiuidualium ${ }^{35}$. Esta percepción de las intenciones individuales se logra mediante la aprehensión de la naturaleza universal que corresponde al objeto que es percibido en una instancia concreta. Esto significa que, cuando Pedro ve una casa, él implícitamente la ve como perteneciente a una clase de objetos, es decir a la clase de las casas. Digo 'implicitamente', porque, para que la vea como tal, no requiere de un acto de reflexión consciente acerca de la naturaleza de la casa. No obstante, para llegar al conocimiento perceptivo de la casa, es necesario que se haya adquirido previamente una noción racional acerca de lo que las casas son en general. Si este conocimiento racional de fondo no existiera, no se llegaría a ningún conocimiento perceptivo de casas.

Por ende, no es necesario que exista en los procesos de la percepción humana un juicio intelectivo conceptual y consciente, ya que Tomás sugiere que las reacciones del aparato perceptivo son de tipo disposicional, de manera que cada vez que un objeto $x$ es visto, se actualiza el conocimiento de fondo $z$, que de hecho puede incluir nociones universales, y que corresponden al objeto $x$. Aunque la relación del conocimiento de fondo con un objeto particular no es consciente, suele Tomás usar el verbo iudicare para referirse a la actividad de juntar, aprehender y discernir las intenciones. Sin embargo, usa este término para describir el proceso de la aprehensión sensitiva, y no para referirse al juicio activo acerca de la verdad o falsedad de proposiciones cognitivas ${ }^{36}$. De esta manera se aprecia que Tomás pretende más que nada establecer criterios para una psicología de la percepción, en la cual la vis cogitativa depende participativamente de las estructuras subyacentes del intelecto, el cual, claro está, puede conceptualizar lo que se percibe.

35 TOMÁs DE AQUINo: In De An. II; 13 p. 121, 196.

36 Véase TellkaMP, J. A.: "Actitudes proposicionales y conocimiento sensible en Tomás de Aquino", Revista Española de Filosofia Medieval IV (1997), pp. 87-102. 
Una razón para sostener el vínculo entre las actividades de la percepción y del intelecto se encuentra en el supuesto que las capacidades inferiores participan en las superiores o, para decirlo de otra manera, que las facultades inferiores dependen de las superiores en cuanto a su alcance cognitivo. Por ello es necesario que una facultad sensitiva participe directamente en el intelecto, y esta facultad es la vis cogitativa ${ }^{37}$.

\section{Iudicium sensus}

Como se ha visto, la percepción de objetos físicos, tanto al nivel de los sensibles propios como de los accidentales, realiza una función de discernimiento. Así, lo blanco es discernido de lo negro y lo pequeño de lo grande. El problema que me guió hasta ahora es el papel de las funciones cognitivas, racionales y no racionales, en la formación de un conocimiento perceptivo complejo e intencional. Para concluir con este acercamiento, trataré de dar algunas indicaciones sobre el juicio perceptivo y su relación no necesaria con los procesos racionales y conceptuales.

El verbo iudicare suele ser utilizado para referirse al discernimiento perceptivo. La interpretación moderna $\mathrm{y}$ contemporánea tiende automáticamente a asumir que los actos de juzgar acerca de los objetos y estados en el mundo son conceptuales. Esta interpretación puede ser contestada con las teorias previamente explicadas.

Tomás de Aquino, por ejemplo, en Summa Theologica I q. 78 a. 4 ad 2 supone que todo sentido exterior tiene un objeto propio: la función del sensus proprius consiste, por tanto, en captar tipos idénticos de datos sensoriales (colores, sonidos) y distinguir entre

37 TOMÄs DE AQUINo: In De An. II, 13 p. 121sq., 195-201: “[...] Dicitur etiam ratio particulares eo quod est collatiua intentionum indiuidualium sicut ratio uniuersalis est collatiua rationum uniuersalium, nichilominus tamen he cuis est in parte sensitiva, quia uis sensitiua in sui suppremo participat aliquid de ui intellectiua in homine, in quo sensus intellectui coniungitur [...]". 
ellos. Otra propiedad del aparato sensitivo, como se ha dicho, reside en distinguir entre los objetos propios de diferentes tipos, como por ejemplo entre blanco y amargo. Por último se le adjudica un juicio al nivel de los sensibles accidentales que refieren a los atributos connotacionales de los objetos sensibles. De esta manera se puede decir que el concepto de iudicium no tiene un sentido único, sino que aplica a muchos procesos de discernimiento y distinción en lo sensible. En cuanto a los sensibles propios, el juicio de los sentidos no yerra $^{38}$, mientras que con respecto a los sensibles comunes y accidentales esto puede ser el caso $^{39}$.

El verbo iudicare lleva, desde el punto de vista de una lectura moderna, a una confusión en cuanto que se tiende a suponer que los procesos de la percepción implican una capacidad casi-racional de juzgar activamente sobre proposiciones perceptivas ${ }^{40}$. Sin lugar a duda se puede traducir el verbo iudicare por 'juzgar', pero considero que será menos problemático concebirlo en términos de 'discernir' y 'distinguir'41. Aunque en el Medioevo latino se usaba el verbo discernere en contextos filosóficos, apenas usado en discusiones sobre el conocimiento, donde se usaba más bien el verbo iudicare. Este uso probablemente se relaciona con la recepción de las diferentes traducciones de las obras aristotélicas, y de hecho Guillermo de Moerbeke traduce consistentemente el verbo griego krinein por el latino iudicare ${ }^{42}$.

A mi manera de ver, el problema principal de una interpretación 'racionalista' de iudicare consiste en el peligro de romper la unidad explicativa de los procesos perceptivos y a fortiori de negar la existencia de un juicio o 'pensamiento' animal en relación con

${ }^{38}$ TOMÁS DE AQUINo: In IV Sent. d. 10 q. 1 a. 4 ad 3

${ }^{39}$ TOMÁs DE AQUINo: De Ver. q. 1 a. 11 c.

${ }^{40}$ Asi por ejemplo HAYEN, A.: L'intentionnel dans la philosophie de Saint Thomas, Paris/Bruxelles: Desclée de Brouwer 1954.

41 Véase el trabajo de Ebert, Th.: "Aristotle on What Is Done in Perceiving", Zeitschrift für philosophische Forschung XXXVI (1983), pp. 181 - 198.

42 TOMÁs DE AQUINO: Su Theol. I q. 78 a. 4 ad 2: "[...] Sensus proprius iudicat de sensibili proprio, discemendo ipsum ab aliis quae cadunt sub eodem sensu, sicut discernendo albo a nigro vel a viridi". 
procesos y contenidos perceptivos. El juicio animal, claro está, no involucra la capacidad de formar proposiciones, tal como lo exige la tradición racionalista ${ }^{43}$.

En lo que sigue quisiera aducir algunos argumentos para interpretar el verbo iudicare en el sentido de los verbos 'distinguir' o 'discernir', los cuales, como se ha dicho, parecen ser menos problemáticos desde el punto de vista epistemológico. El efecto de una separación semántica de los actos de juzgar y los actos de discernir es claro: las percepciones no son actos de opinión (opinio, doxa). Las siguientes son algunas diferencias entre los actos juzgar/opinar (en el sentido racionalista) y las actividades de discernir ${ }^{44}$.

1. 'Discernir' es un verbo de éxito, mientras que un juicio erróneo sigue siendo un juicio.

2. Algo puede ser distinguido de manera clara, pero no de manera verdadera y falsa. Criterios de verdad, sin embargo, aplican a juicios.

3. 'Juzgar' es un verbo proposicional, 'discernir' es un verbo cognitivo. Esto significa que 'discernir' no depende de proposiciones reconocidas como verdaderas, como lo es el caso en los juicios, que suelen ser formados de manera discursiva a partir de una proposición para llegar a otra. 'Discernir' refiere directamente al objeto del que algo se discierne. Un juicio, en cambio, se relaciona con la verdad de una proposición.

\footnotetext{
${ }^{43}$ Para una presentación corta del concepto iudicium en Pedro Hispano y Tomás de Aquino véase Nuchelmans, G.: Theories of the Proposition, Amsterdam/ Londres: North-Holland Publishing Co. 1973, p. 189ss. Cf. también Hayen op.cit. pp. 127. 132 y GARCEAU, B: judicium. Vocabulaire, sounces, doctrine de saint Thomas d'Aquin, Montreal/Paris: Vrin 1968, pp. 24lss. HASSE op.cit. p. 136 y AVICENA: De an. $I V, 1$ p. 8, 97-99: "Et haec virtus [i.e. aestimativa - J.A.T.] sine dubio consistit in nobis; quae es domina, iudicans in animali iudicium non definitum sicut iudicium intellectuale [...]".

44 Véase EBERT op.cit. p. 184 en relación a Aristóteles. También MICHON op.cit.
} 
4. 'Discernir' refiere a una actividad delimitada temporalmente, mientras que 'juzgar' no refiere a un marco temporal.

5. Las propiedades que en su mayoria aplican a 'discernir', también aplican a verbos como 'oír', 'ver', etc. La propiedad común de estos verbos consiste en que poseen un objeto cognitivo directo, es decir que refieren a procesos perceptivos, pero también a aquellas actividades que se basan en ellos, como 'observar' o. 'escuchar'. 'Discernir' implica la actualización cognitiva de una facultad o potencia.

\section{A manera de conclusión}

A pesar de las diferencias entre la vis aestimativa/cogitativa y los diferentes grados de complejidad de los conocimientos animal y humano, el 'isomorfismo estructural parece constituir una interpretación viable para dar cuenta del conocimiento sensible. Las razones para ello no radican en que el conocimiento humano se base en una estructura cognitiva racional subyacente y el de los animales no, sino porque tanto los animales superiores como los seres humanos comparten los mismos mecanismos fisiológicos. La distinción moderna entre seres humanos, como seres cognitivos, y animales, como una suerte de máquinas, es una distinción que tanto Avicena como Tomás habrian rechazado rotundamente. 0 ; para decirlo con C. Michon, quien habia citado al principio, deberiamos aceptar la existencia de una forma compleja de conocimiento para poder explicar el comportamiento animal, pero también deberíamos aceptar que la capacidad propia del ser humano de hablar, influye en la percepción, aunque no la condicione de manera consciente ${ }^{45}$.

${ }^{45}$ MiCHON op.cit. 336s. 
Copyright of Tópicos. Revista de Filosofía is the property of Universidad Panamericana and its content may not be copied or emailed to multiple sites or posted to a listserv without the copyright holder's express written permission. However, users may print, download, or email articles for individual use. 